\begin{tabular}{|l|l|l|l|}
\hline Eiszeitalter u. Gegenwart & Band 25 & Seite 132-140 & Öhringen/Württ., 31. Oktober 1974 \\
\hline
\end{tabular}

\title{
Fossile Riesen-Eiskeilnetze am Niederrhein
}

\author{
Von Winfried Golte und Klaus Heine, Bonn
}

Mit 8 Abbildungen

$\mathrm{Zus}$ a m menfassung. Auf niederrheinischen Terrassenschottern finden sich in zum Teil regelmäßigen Abständen taschenförmige Gebilde mit einer durch Eisenoxyde und -hydroxyde auffällig gefärbten Füllung von Feinmaterial. Sie können bis zu $6 \mathrm{~m}$ breit werden und bis etwa $3 \mathrm{~m}$ in die Tiefe reichen. Bisher wurden sie als Würge- und Taschenböden gedeutet. Es wird gezeigt, daß diese Gebilde netzartig miteinander in Verbindung stehen und Zeugen fossiler Eiskeilnetze darstellen. Ihre Bildung erfolgte sehr wahrscheinlich nur während der Rißeiszeit im unmittelbaren Vorfeld des Inlandeises.

$\mathrm{Su} \mathrm{m} \mathrm{m}$ a r y. On the gravel terraces of the Lower Rhine area one can find in more or less regular distances forms resembling pockets which show a conspicious coloured (Fe-Oxides and -Hydroxides) filling of fine materials. They can get a width up to $6 \mathrm{~m}$ and a depth of $3 \mathrm{~m}$. Till today they have been interpreted as cryoturbations. Like a network these forms are connected with one another and represent fossil ice-wedge polygons. Probably they came into existence during the Riss glaciation, right in the surroundings of the inland ice.

\section{Einleitung}

In den ausgedehnten Schotterablagerungen des Niederrheingebietes finden sich immer wieder in mehr oder weniger regelmäßigen Abständen auffällige taschenartige Gebilde, deren Füllung überwiegend aus Feinmaterial besteht. Von den Kiesgrubenarbeitern werden derartige Bildungen, die sich schon durch ihre vorherrschend intensiv rostbraune Verfärbung von den umgebenden Schottern abheben und durch ihre zementartig verbackene Beschaffenheit den Kiesabbau erschweren, meist als „Klei-Köppe“ bezeichnet. Vor nunmehr 50 Jahren wurden sie erstmalig von STEEger (1925) aus der Krefelder Mittelterrasse beschrieben. STEEgER (1944) selbst hat seine Beobachtungen noch einmal im „Klimaheft“ der Geologischen Rundschau zusammengestellt. Zuletzt wurden sie von Mertens (1971)
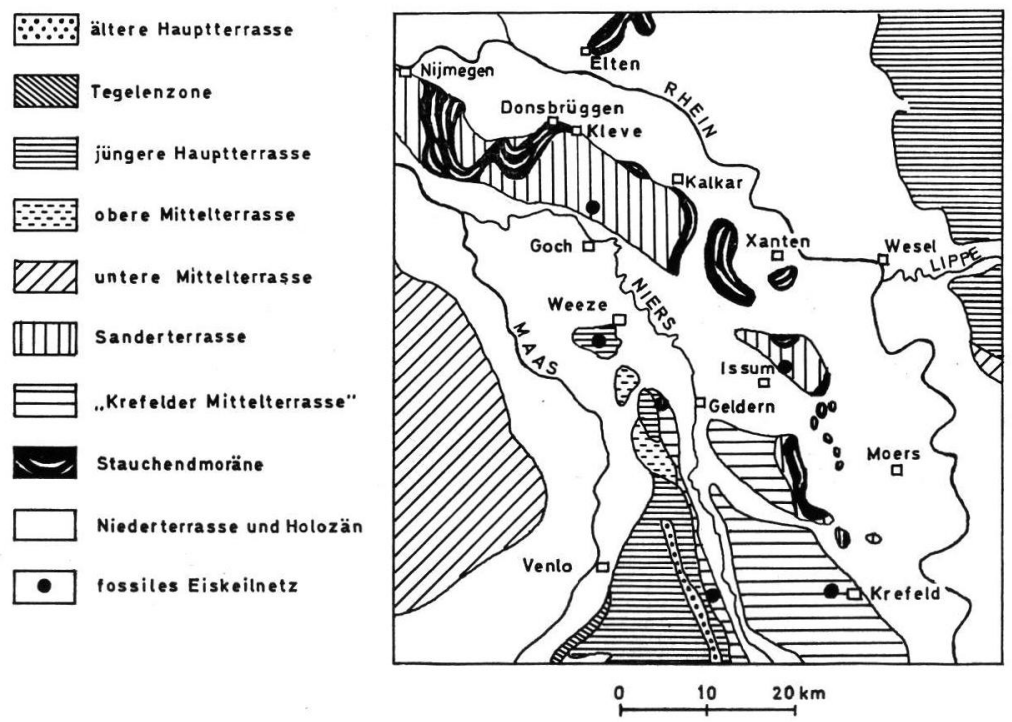

Abb. 1. Geomorphologische Skizze des unteren Niederrheins (nach Wagner 1968). 
als „kaltzeitliche Bodenfrosterscheinungen“ beschrieben. Wie vor ihm STEEGER erklärt auch Mertens die Taschen als kryoturbate Bildungen, die sich von den weiter verbreiteten Verwürgungen lediglich durch den hohen Grad der Materialsortierung unterschieden. Ebenso haben die Verfasser selbst (1971) bei der Beschreibung besonders schön ausgebildeter Taschen vom Südrand der Weezer Hees diese als Kryoturbationen gedeutet.

Die bisherigen Deutungen waren jedoch sehr unbefriedigend, und stets blieb schwer vorstellbar, wie durch Kryoturbation derart regelmäßig geformte Gebilde entstanden sein sollen.

Weitere Beobachtungen und Untersuchungen im Gelände haben ergeben, daß es sich in Wirklichkeit um fossile Riesen-Eiskeilnetze handelt ${ }^{1}$ ).

\section{Die Riesen-Eiskeilnetze am Niederrhein}

\subsection{B es chreibung}

Abbildung 2 zeigt eine Tasche vom Nordrand der Weezer Hees (Kiesgrube Venhoven). Aus ihrem Aufbau (Abb. 3) werden die charakteristischen, immer wiederkehrenden Merkmale ersichtlich.

Den Inhalt der Tasche bildet ein schlecht sortiertes tonig-schluffig-sandiges Material mit vereinzelten Geröllen. Die vorherrschende Farbe ist ein intensives Rostbraun (Munsell 5 YR 5/8). Darin befinden sich Schlieren und Bänder von weißlich-grauer Farbe (Munsell 7.5 YR N8/), die zum Teil in ihrer Anordnung die Form der Tasche nachzeichnen. Korngrößenanalysen ergeben, daß die unterschiedliche Färbung unterschiedliche Kornverteilungen widerspiegelt (Tab. 1). Das weiß-graue Material ist stets feiner als das rostbraun gefärbte; der Ton-, Schluff- und Sandanteil beträgt 22,5\%/21,8\%/55,7 \% für das erstere und 12,7\% / 10,6\% / 76,7\% für das letztere. Die weiß-grauen Schlieren werden häufig von dünnen dunkelbraunen Eisen-/Manganoxyd- und -hydroxydkrusten umgeben. An der Basis der Tasche ist eine feine Bänderung (Lamellierung) nach Korngröße und Farbe zu beobachten, die dem gebogenen Verlauf der Taschensohle folgt (Abb. 8). Der Tonanteil einer Mischprobe der teilweise nur millimeterstarken Bändchen betrug 30,3\% sand fehlen nahezu vollkommen (Tab. 1, Probe 5). Im mittleren und oberen Teil der Tasche, wo die hellen Schlieren mehr oder weniger senkrecht angeordnet sind und dabei zuweilen eine Marmorierung hervorrufen, wird eine ähnliche Korngrößenverteilung erreicht (Probe 3). Gerölle bis zu Walnußgröße finden sich unregelmäßig im gesamten Feinmaterial verstreut. Gestreckte Gerölle lassen eine Einregelung der Längsachsen im Sinne der Anordnung der hellen Schlieren erkennen.

Stets sind die Taschen in fluvial geschichtete Schotter eingesenkt. In ihrer unmittelbaren Umgebung allerdings ist die ursprüngliche Schichtung der Schotter in charakteristischer Weise gestört. Unter der Taschensohle sind die mehr oder weniger horizontal gelagerten Sedimente nach unten eingebeult. Die Anordnung der einzelnen Gerölle freilich zeigt hier trotz erhalten gebliebener Schichtung, daß sie etwas bewegt worden sein müssen. Zu beiden Seiten der Tasche sind die fluvialen Schotter am stärksten gestört. Bis in etwa halbe Höhe sind Geröllschichten aufgebogen. Schichtungsmerkmale sind im oberen Teil nicht mehr zu erkennen. Es fällt auf, daß hier Geröllpackungen bis unmittelbar unter den ackerbaulich genutzten Boden reichen. Von den Seiten her sind Kiesnester und -bänder über die randlichen Teile der Tasche gezogen. Die Deckschicht über dem zentralen Teil der Tasche hingegen besteht aus einem gut sortierten, schichtungslosen Mittel- bis Feinsand (Proben 4 und 6) von heller fahlbrauner Farbe (Munsell 10 YR 7/4) mit nur wenigen eingestreuten

1) Obwohl sich mit dem Begriff "Tasche“ allzu leicht die Vorstellung kryoturbater Entstehung verbindet, soll er im Folgenden beibehalten werden, weil er besser als jeder andere Form und Inhalt beschreibt. 


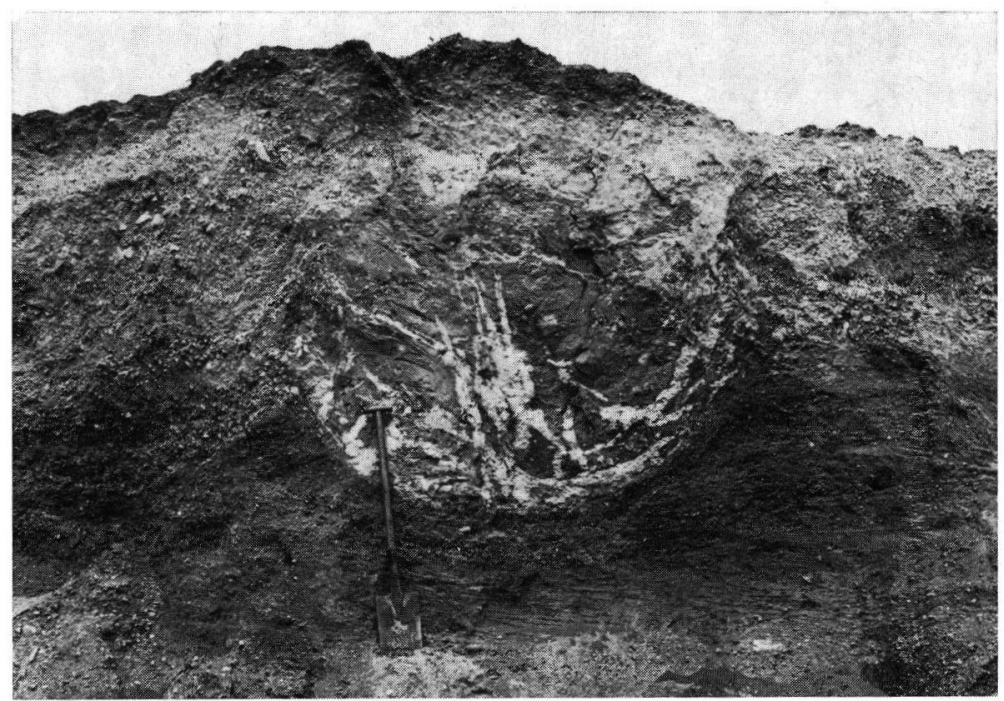

Abb. 2. Fossiler Eiskeil in Schottern der jüngeren Hauptterrasse in der Weezer Hees (Nordwand der Kiesgrube Venhoven, Weeze-Baal). Zur Erläuterung s. Abb. 3.

Geröllen. Gerölle in unmittelbarer Umgebung der Feinmaterialfüllung zeigen vielfach eine tangentiale Einregelung ihrer Längsachsen. In den seitlich über die Tasche herausragenden Geröllpackungen stehen sie häufig senkrecht.

Tab. 1. Korngrößenanalysen verschiedener Proben aus Taschenfüllungen (Prozentwerte) (zur Herkunft der Proben s. Abb. 3 u. 6. Die Proben 5 u. 6 entstammen im Text nicht erwähnten "Taschen")

\begin{tabular}{l|r|r|r|r|r|r|r|r}
\hline $\begin{array}{l}\text { Korn- } \\
\text { größenklassen }\end{array}$ & 1 & 2 & 3 & 4 & 5 & 6 & 7 & 8 \\
\hline $2-1 \mathrm{~mm}$ & 0.8 & 0.5 & 1.0 & 0.3 & - & 2.7 & 2.4 & 3.4 \\
$1-0.63 \mathrm{~mm}$ & 0.6 & 1.1 & 0.5 & 0.4 & - & 1.7 & 1.7 & 2.7 \\
$0.63-0.2 \mathrm{~mm}$ & 19.7 & 52.1 & 9.2 & 52.7 & 0.2 & 55.9 & 47.4 & 33.7 \\
$0.2-0.1 \mathrm{~mm}$ & 12.2 & 15.0 & 16.0 & 24.1 & 1.6 & 15.4 & 17.6 & 13.0 \\
$0.1-0.063 \mathrm{~mm}$ & 22.4 & 8.0 & 28.8 & 9.4 & 9.8 & 6.0 & 6.8 & 8.2 \\
$60-20 \mu$ & 17.0 & 8.8 & 16.5 & 8.4 & 35.7 & 12.9 & 11.4 & 18.0 \\
$20-6.3 \mu$ & 4.8 & 1.8 & 5.0 & 0.1 & 21.6 & 3.0 & 3.2 & 4.7 \\
$6.3-2 \mu$ & - & - & - & - & 0.8 & - & - & - \\
$<2 \mu$ & 22.5 & 12.7 & 23.8 & 4.6 & 30.3 & 2.4 & 9.5 & 16.3 \\
\hline & $100 .-$ & $100 .-$ & $100 .-$ & $100 .-$ & $100 .-$ & $100 .-$ & $100 .-$ & $100 .-$
\end{tabular}

Bereits Steeger $(1925,1944)$ hat durch Nachgraben feststellen können, daß die Taschen sich meist „talartig mehrere Meter weit fortsetzen“ $(1944,522)$. In der Tat handelt es sich, wie eigene Grabungen, Bohrungen und Beobachtungen gezeigt haben, bei den $\mathrm{Ta}-$ schen um Querschnitte langgestreckter Gebilde. An verschieden gerichteten Wänden der untersuchten Aufschlüsse waren daher nicht nur Quer-, sondern auch Längs- und Schrägschnitte zu finden. $\mathrm{Da}$ diese meist eine weniger regelmäßige Anordnung der Sedimente 


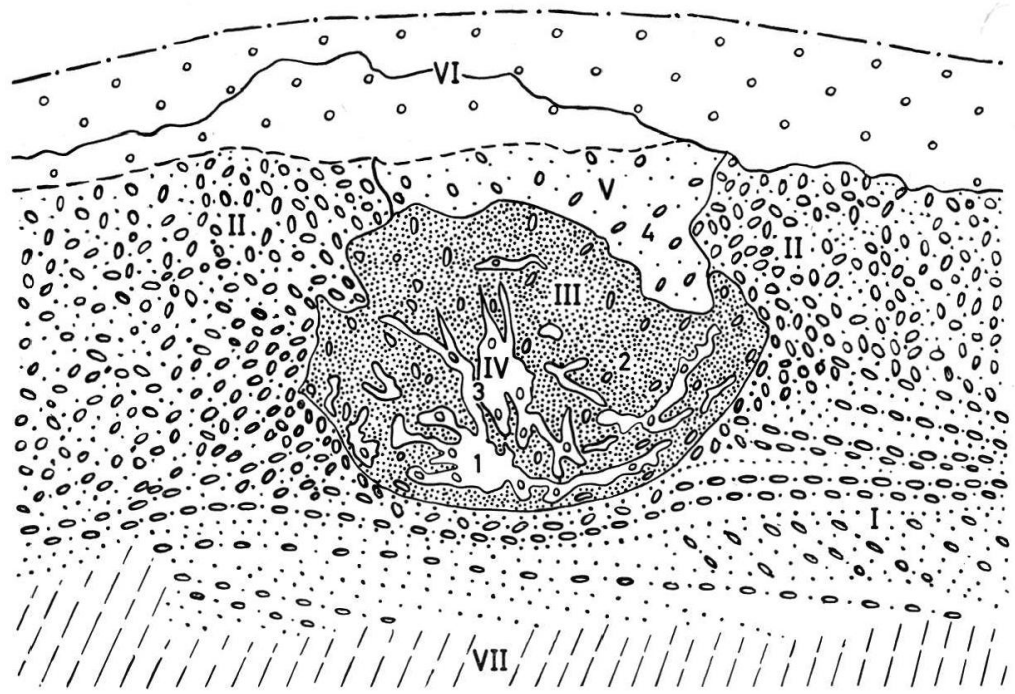

Abb. 3. Profil des in Abb. 2 gezeigten fossilen Eiskeils. Die rö mis che $\mathrm{n}$ Ziffern bedeuten: $\mathrm{I}=$ ungestörte Schotter der $\mathrm{jHT}$; II = seitlich zusammen- und aufgepreßte Geröllpackungen; III = rostbraune, tonig-schluffig-sandige Feinmaterialfüllung mit eingestreuten Geröllen; IV = weißgraue Schlieren von Feinmaterial mit eingestreuten Geröllen; V = fahlbrauner Mittel- bis Feinsand mit eingestreuten Geröllen; VI = rezenter Boden (Podsol) auf Sand mit eingestreuten Geröllen, größtenteils abgeschoben; VII = Böschung. - Die a r a b i s ch e n Ziffern bezeichnen die Entnahmestellen der Proben 1-4 in Tab. 1.

nach Farbe und Textur erkennen lassen als die Querschnitte, dürfte hier ein Hauptgrund für die bisherigen Fehldeutungen der Gebilde als kryoturbate Formen liegen. Unzweifelhaft aber sind diese in verschiedenster Weise angeschnittenen Gebilde nur Teile eines zusammenhängenden Netzes. Dafür sprechen vor allem auch die Abstände der Taschen in den fluvialen Schottern. Diese sind teilweise auffallend regelmäßig $(6-8 \mathrm{~m})$, während an anderen Wänden ein und derselben Kiesgrube Abstände bis über $30 \mathrm{~m}$ einerseits und von nur wenigen Dezimetern andererseits beobachtet wurden. „Zwillingstaschen“ zeigen an, daß ein Knotenpunkt des Netzes angeschnitten ist. Besonders eindrucksvoll wirken die rotbraunen Taschen dort, wo sie mit weiten Abständen in helle, gut geschichtete Sedimente eingelagert sind, beispielsweise in dem Kalksandstein-Werk Issum oder am Südwestrand der Weezer Hees (vgl. Abb. 4).

Aus den Beobachtungen ergibt sich eine recht unterschiedliche Maschenweite des Netzes. Neben sehr unregelmäßigen polygonalen Grundrissen müssen ungefähr gleich große und mehr oder weniger rechteckige Maschen gebildet worden sein.

\subsection{Deutung}

Nach den Befunden kann kein Zweifel daran bestehen, daß es sich bei den beschriebenen Formen um die Zeugen von Riesen-Eiskeilnetzen handelt. Die Formen lassen sich nur verstehen, wenn man sie einerseits aus der Eiskeilbildung selbst und andererseits aus dem Zusammenbruch des vorhandenen Eiskeilnetzes erklärt. Das frühere Vorhandensein von Eiskeilen belegen:

a) Die Form der Taschen (Abb. 5). Schon STEeger (1944, 522), der der richtigen Deutung durch genaue Beobachtungen recht nahe gekommen ist, spricht von „taschen-, kessel-, trichter-, trauben-, birnen-, trog- oder wannenförmigen“ Querschnitten. Beson- 


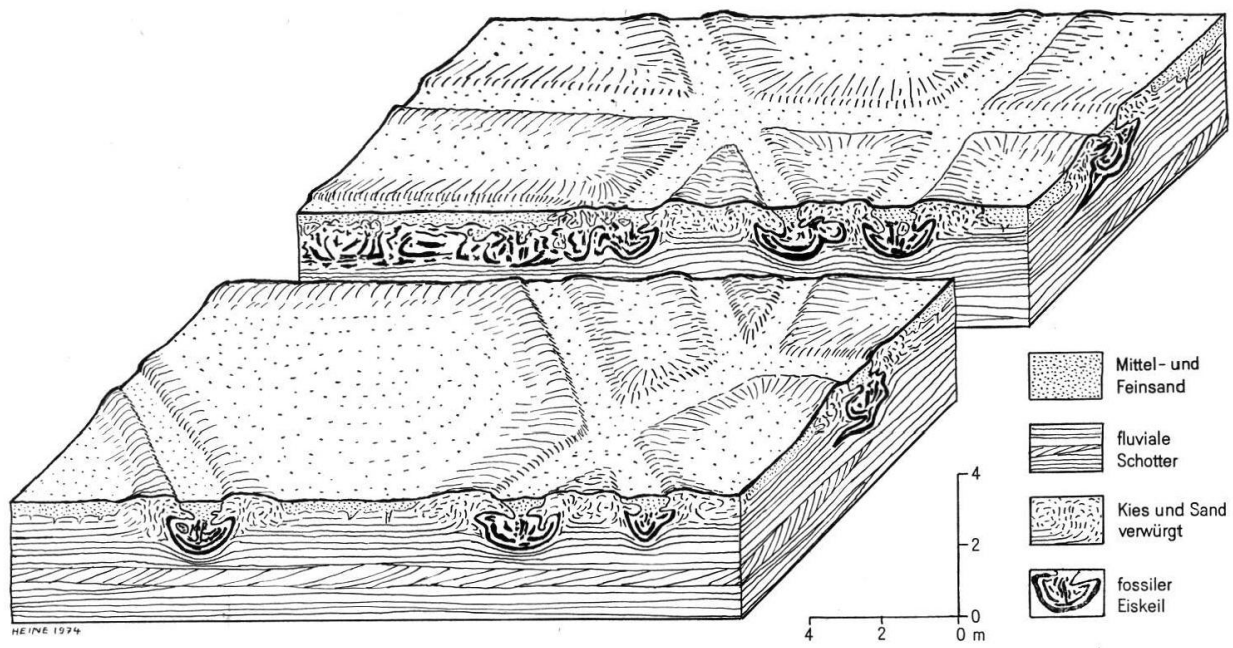

A.bb. 4. Rekonstruktion eines fossilen Eiskeilnetzes in der Weezer Hees. Der 25-50 cm mächtige rezente (Podsol-)Boden auf Sand ist in dem Blockdiagramm abgedeckt.

ders instruktiv sind Abbildung und Beschreibung der "Ampulle von Krefeld“ (1944, 528 f.). Formen wie diese und andere, die dem herkömmlichen Verständnis der Keilform am besten entsprechen, sind eindeutige Belege für unsere Eiskeil-Theorie und wären durch Verwürgung bzw. Materialsortierung nicht zu erklären.

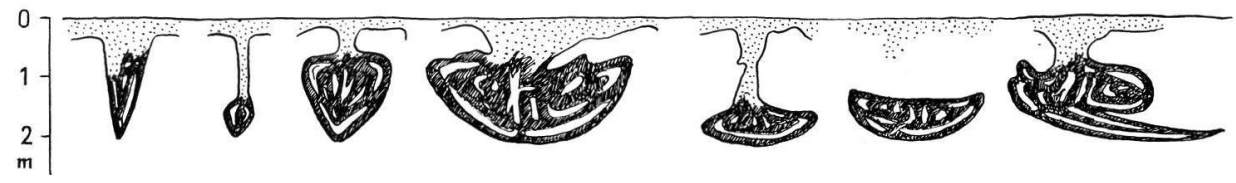

Abb. 5. Auswahl verschiedener "Taschen“querschnitte.

b) Die seitlich aufgepreßten und gestörten G e r ö 11 p a ck u n g e n (vgl. Abb. 2 u. 3). Das lamellenartige Wachsen und Verbreitern eines Eiskörpers (Eiskeil) bewirkt durch starken seitlichen Druck auf die fluvial geschichteten Schotter deren Stauchung und Aufpressung nach oben. Sehr anschauliche Abbildungen lebender Eiskeile, welche diese Vorgänge illustrieren, finden sich bei Troll (1944, 635), WAshburn (1973, 36 u. 94-96) und Péwé et al. (1973, 17-18). Luftaufnahmen rezenter Eiskeilnetze (z. B. PÉWÉ et al. 1973, 18) zeigen in Übereinstimmung mit früheren Beschreibungen (Troll 1944, 637), daß sich die Lage der Eiskeile im Dauerfrostboden an der Oberfläche durch ein Mikrorelief abzeichnet. Uber den Eiskeilen befinden sich flache Rinnen. Diese werden begrenzt durch schmale Wälle, die steil zu den Rinnen und flach konkav gegen das Zentrum der durch sie gebildeten Polygone einfallen. Reste jener rahmenden Wälle sind am Niedrrhein in Gestalt der seitlich aufgepreßten Geröllpackungen noch gut zu erkennen.

c) Der von einem Eiskeil ausgehende seit liche Schub bzw. Druck kann aufgrund eines glücklichen Fundes im vorliegenden Zusammenhang anschaulich belegt werden. Eine der in der Kiesgrube $1 \mathrm{~km}$ westlich des Ortes Wemb in der Weezer Hees aufgeschlossenen Taschen zeigte genau an ihrer Basis, d. h. unmittelbar unter der Feinmaterialfüllung, einen durch starken seitlichen Druck auf zwei Schichtflächen auseinandergeschobenen paläozoischen Sandstein (Abb. 6). Nach dem Herauslösen aus der Wand ließen sich die drei größeren Platten und die kleineren Bruchstücke ohne Schwierigkeit wieder zur 


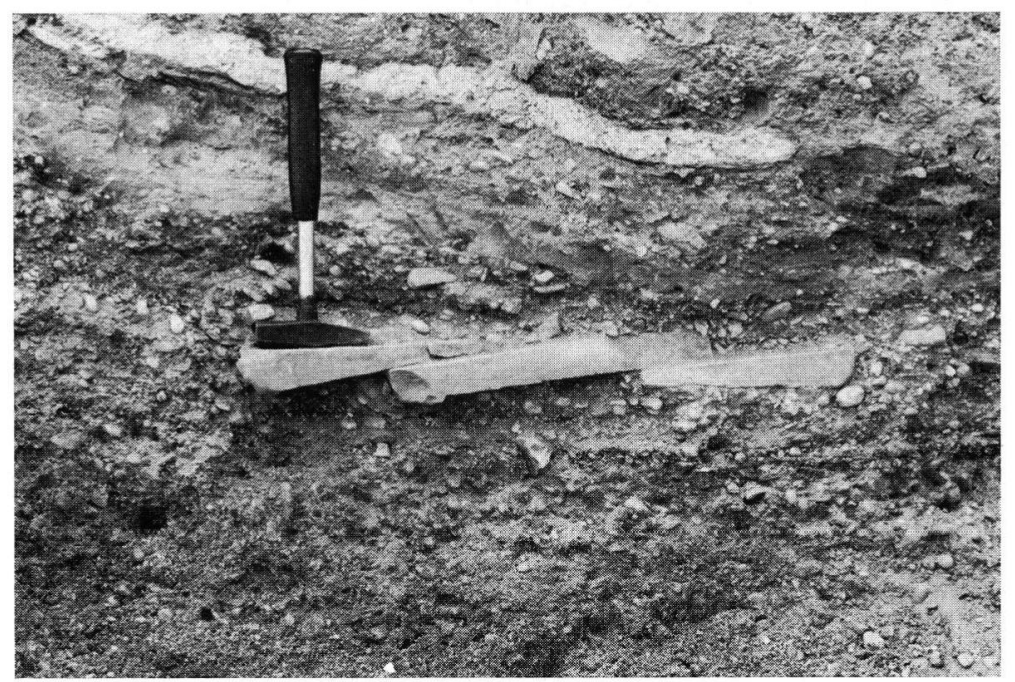

Abb. 6. Auseinandergedrückter paläozoischer Sandsteinblock an der Basis eines fossilen Eiskeils in der Weezer Hees. Aus dem von links oben hereinziehenden weißgrauen Feinmaterialband stammt Probe 8 der Tab. 1, aus dem darüberliegenden rostbraunen Material Probe 7. Weitere Erl. im Text.

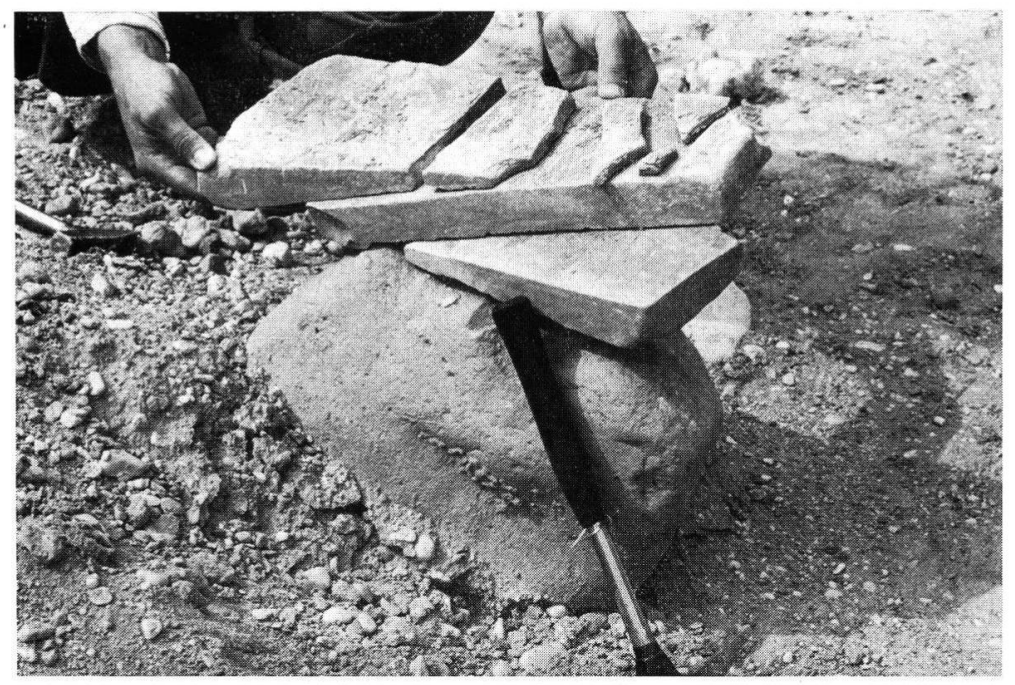

Abb. 7. Der in Abb. 6 in situ befindliche Sandsteinblock so wieder zusammengefügt, daß Schichtflächen und Bruchstellen gut sichtbar sind. Weitere Erl. im Text.

ursprünglichen Form zusammenfügen (Abb. 7). Der mit seiner Längsachse quer zur Richtung des Eiskeils liegende Block wog $11 \mathrm{~kg}$ und maß $37 \mathrm{~cm}$ Länge, $18 \mathrm{~cm}$ größte sowie $9 \mathrm{~cm}$ kleinste Breite. Die Gesamthöhe betrug $8 \mathrm{~cm}$. Offensichtlich stak er mit der Unterseite starr im Schotter, während sein oberer Teil durch den Druck des sich erweiternden Eiskeils erfaßt und in zwei Platten seitlich um einen Gesamtbetrag von $29 \mathrm{~cm}$ verschoben wurde (Länge über alle drei Platten daher $66 \mathrm{~cm}$ ). Der auf diese Weise in drei (große) Teile zerlegte Block bot den Eindruck dachziegelartiger Überlappung. Die Lagerungsverhältnisse ebenso wie die Tatsache, daß an den Stellen stärkster Beanspruchung kleinere 
Stücke abgebrochen waren, schließen vollständig aus, daß er so bereits primär sedimentiert wurde. Auch an anderer Stelle konnten wir - wenn auch nicht entfernt so anschaulich wie im beschriebenen Falle - an der Untergrenze von Eiskeilen auseinandergedrückte Steine beobachten.

d) Das E is keilnetz entspricht voll und ganz den in der Literatur beschriebenen Formen (Leffingwell 1915; Troll 1944; Péwé et al. 1969; PÉwé 1973).

e) Aufgrund der heutigen Kenntnis des eiszeitlichen Klimas in Mitteleuropa waren am Niederrhein mindestens zeitweilig die klimatischen Voraussatzungen für die Bildung eines Eiskeilnetzes gegeben.

Der Zusammenbruch des vorhandenen Eiskeilnetzes wird durch folgende Beobachtungen belegt:

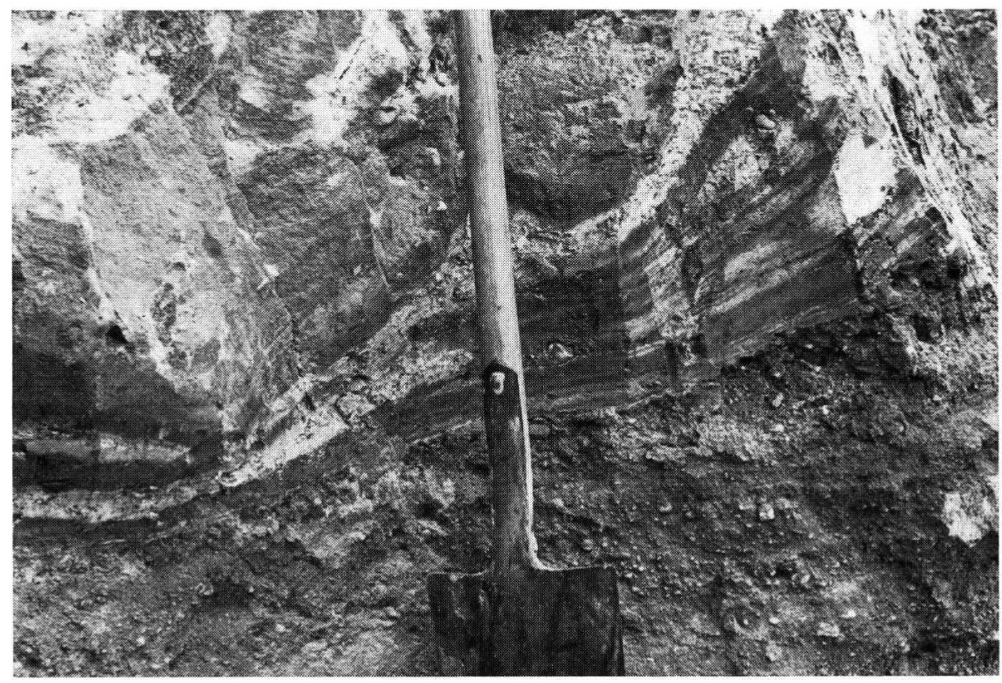

Abb. 8. Lamellierung im unteren Abschnitt eines fossilen Eiskeils in der Weezer Hees, das sukzessive Ersetzen des abtauenden Eiskeils durch Feinmaterial zeigend (Kiesgrube Venhoven, Weeze-Baal).

a) Der schalige $\mathrm{Aufb}$ a u der Füllung der Taschen, kenntlich vor allen Dingen an der unterschiedlichen Färbung (Abb. 8), ist besonders im unteren Teil der Taschen zu finden und dem sukzessiven Ersetzen des abschmelzenden Eises durch feinkörnige Sedimente zuzuschreiben.

b) Die Materialsortierung ist sehr unterschiedlich (Tab. 1). Obwohl die Korngrößenverteilung innerhalb der einzelnen Schalen bzw. Schlieren sehr heterogen ist (mehrphasiges Sediment), wie die Proben zeigen, heben sich die Schalen doch recht deutlich voneinander ab. Der größte Teil der Taschenfüllung besteht aus Mittel- bis Feinsand und entstammt den sandigen Decksedimenten (vgl. Proben 4 und 6). Bei der Auffüllung des Eiskeils wurcen außerdem in unterschiedlichem Maße schluffige und tonige Bestandteile eingewaschen. Die gleichen Beobachtungen machten PÉWÉ et al. (1969) und PÉWÉ (1973) an fossilen Eiskeilen in Alaska.

c) Die Ein regelung der in die Füllung eingestreuten Gerölle spiegelt den Vorgang des Ersetzens des Eises durch ein- und nachdringende Sedimente wider. Im zentralen oberen Teil der Tasche beispielsweise stehen die Längsachsen mehr oder weniger vertikal.

d) Die von den aufgepreßten Geröllpackungen an den Seiten gegen das Zentrum der Tasche ziehenden K i es n e ste r - und - bänd e r belegen sehr eindrucksvoll das end- 
gültige Kollabieren des Eiskeils. Ein Teil des Materials der die Eiskeile rahmenden Wälle gelangte im Gefolge der Abschmelzprozesse in die dabei sich vertiefenden Rinnen des Eiskeilnetzes.

\section{Diskussion der Ergebnisse}

Die vorgetragene Deutung der taschenartigen Gebilde als Zeugen eines fossilen RiesenEiskeilnetzes steht in Gegensatz zu den bisherigen Versuchen, sie als Ergebnisse von Verwürgung und Materialsortierung zu erklären. Uns ist keine Arbeit aus Europa bekannt, die ähnliche Gebilde beschreiben und übereinstimmend deuten würde. Doch liegen aus Nordamerika vor allem die gründlichen Arbeiten des Eiskeilspezialisten PÉwÉ vor. Besonders aufschlußreich für einen Vergleich ist die von ihm in Gemeinschaft mit CHURCH und Andresen (PÉwÉ et al. 1969) verfaßte Abhandlung über Ursprung und paläoklimatische Bedeutung von Eiskeilnetzen des Donnelly-Dome-Gebietes in Alaska. Die darin beschriebenen fossilen, Wisconsin-zeitlichen Eiskeilnetze weisen die gleichen Merkmale auf wie die von uns im Niederrheingebiet untersuchten.

Nach PÉwé (1973) sind Eiskeile und Eiskeilnetze neben Pingos und Blockgletschern die einzigen sicheren Zeugen für einen Dauerfrostboden. Netze wie die vom Niederrhein beschriebenen entstehen, wenn infolge allgemeiner Erwärmung die Eiskeile langsam schmelzen und der Dauerfrostboden von oben her allmählich verschwindet. Mit dem Abschmelzen des oberen Eiskeils werden zunächst die über dem Netz verlaufenden Rinnen vertieft. In ihnen sammelt sich abfließendes Wasser, welches bis auf das abschmelzende Eis hindurchsickert und Feinmaterial mit sich führt. Beim weiteren Herabschmelzen des Eiskeils und Tauen der umgebenden Sedimente, die dadurch ihr Bindemittel (Eis) verlieren, neigen die Sedimente dazu, in dem vom Eiskeil freigegebenen Bereich hinein zu kollabieren. Diese Vorgänge sind besonders an grobe, sandige Schotter gebunden (vgl. PÉwé 1973, 19). Kollabieren des rahmenden Materials und Infiltration von Feinmaterial von oben und von den Seiten führen zu der für die beschriebenen Taschen charakteristischen Heterogenität der Füllung.

Mit der Korngrößenverteilung hängt die intensiv rostbraune bzw. weißgraue Färbung der Taschen zusammen. Das spezifische Porenvolumen der Taschenfüllung bedingt einen hohen Haftwassergehalt im Vergleich zu den umgebenden Schottern. Am höchsten ist der Wassergehalt in den ton- und schluffreichen Lagen am Boden der Taschen. Ahnlich wie in vergleyten Böden sind daher hier Oxydations- und Reduktionsvorgänge besonders intensiv. In den jeweils feinsten ton- und schluffreichen Lagen bedingt reduziertes Eisen die weißlich-graue Farbe, während die gröberen Sedimente infolge leichterer Austrocknung durch Eisenoxyde und -hydroxyde rostbraun gefärbt sind. Zusätzlich dürfte eine gewisse Filterwirkung des Tascheninhalts dazu geführt haben, daß aus dem im gesamten Schotterkörper zirkulierenden Wasser Eisenoxyde und -hydroxyde hier angereichert wurden.

Heute gibt es lebende Eiskeile und Eiskeilnetze nur in Gebieten mit einem zusammenhängenden Dauerfrostboden bei einer Jahresmitteltemperatur von $-7^{\circ} \mathrm{C}$ und darunter (PÉwÉ 1973, 24). Es handelt sich um hochkontinentale Tundren- und Frostschuttklimate. Für die Bildung von Eiskeilnetzen in fluvialen Schottern sind extrem kalte Winter Voraussetzung, da nur dann die Kontraktion des gefrorenen Schotterkörpers groß genug ist, um ein Aufreißen von Frostspalten zu ermöglichen, aus denen später ein Eiskeilnetz hervorgehen kann. Unter dieser Annahme müssen am Niederrhein zeitweilig Jahresmitteltemperaturen von $-6^{\circ}$ bis $-8^{\circ} \mathrm{C}$ geherrscht haben, was einer eiszeitlichen Temperaturabsenkung von mindestens $15^{\circ} \mathrm{C}$ entspricht.

Mit dieser postulierten Temperaturabnahme verbindet sich die Frage nach dem Alter der niederrheinischen Eiskeilnetze. Vieles spricht dafür, daß es sich um rißeiszeitliche Bil- 
dungen handelt. Bisher sind uns keine fossilen Eiskeilnetze auf würmzeitlichen Schotterterrassen am Niederrhein bekannt geworden. Doch auch auf älteren Schottern haben sie nur eine verhältnismäßig begrenzte Verbreitung im Vorfeld der Drenthe-zeitlichen Inlandvereisung. Die Beobachtungen in Nordamerika legen ebenfalls einen Zusammenhang von Eiskeilnetzen und Eisrandlagen nahe (vgl. PÉWÉ 1973). Nach dem stratigraphischen Befund und dem beobachteten Zusammenhang mit der Eisrandlage kommt nur die RißEiszeit (Drenthe-Stadium) als Bildungszeit der niederrheinischen Eiskeilnetze in Frage. Dafür sprechen auch die auf Schottern der unteren Mittelterrasse unter mächtigen würmzeitlichen Lößsedimenten beobachteten Taschen bei Frimmersdorf an der Erft (v. D. BRELIE et al. 1959). Die für die Bildung notwendige Temperaturabsenkung von mindestens $15^{\circ} \mathrm{C}$ gegenüber dem heutigen Jahresmittel ist vermutlich nur im Vorfeld des Drenthe-zeitlichen Inlandeises erreicht worden (erhöhte Albedo der Eismasse, Verdunstungskälte, kalte Fallwinde).

Wenn aber die Eiskeilnetze in Schottern nur während des Drenthe-Stadiums entstanden sein können, dann bedeutet dies, daß die Krefelder Mittelterrasse, auf der sich ebenso wie auf der Sanderterrasse der Bönninghardt Eiskeilnetze ausgebildet haben, Drenthezeitlich ist und damit im Niederrheingebiet die Untere Mittelterrasse des Mittelrheins vertritt. Darauf hat Thome wiederholt hingewiesen (1959 u. frdl. schr. Mitt. vom 17.1.1972).

Abschließend bleibt festzustellen, daß große Eiskeilnetze von der beschriebenen Art fossil wie rezent nur in verhältnismäßig flachen Schotterebenen mit der Möglichkeit starker Wasserdurchtränkung vorkommen. Bei der Bildung von Eiskeilnetzen in anderen Substraten können sicher auch andere Entstehungsbedingungen als die erörterten geherrscht haben. So hat SELzer (1936) aus dem Leinetalgraben ein vermutlich würmzeitliches Eiskeilnetz in Löß beschrieben, das weit entfernt vom entsprechenden Eisrand liegt.

\section{Literatur}

Brelie, G. v. D., Kilpper, K. \& Teichmüller, R.: Das Pleistozän-Profil von Frimmersdorf an der Erft. - Fortschr. Geol. Rheinl. u. Westf., Bd. 4, 179-196, Krefeld 1959.

Golte, W. \& Heine, K.: Zur Deutung eiszeitlicher Ablagerungen und Formen am Niederrhein. Rhein. Heimatpflege, N. F., 180-187, 1971.

LefFingwell, E. DE K.: Ground-Ice Wedges. The dominant Form of Ground-Ice on the North Coast of Alaska. - The Journ. of Geol. 23, Chicago 1915.

Mertens, H.: Kaltzeitliche Bodenfrosterscheinungen im Kreisgebiet. - Heimatbuch d. Kreises Kempen-Krefeld 1972, F. 23, 38-42, Kempen 1971.

PÉwé, T. L.: Ice Wedge Casts and past Permafrost Distribution in North America. - Geoforum 15, 15-26, Braunschweig 1973.

PÉwé, T. L., Church, R. E. and Andresen, M. J.: Origin and paleoclimatic Significance of LargeScale patterned Ground in the Donnelly Dome Area, Alaska. - Spec. Pap. geol. Soc. Am. 109, Boulder/Col. 1969.

Selzer, G.: Diluviale Lößkeile und Lößkeilnetze aus der Umgebung Göttingens. - Geol. Rdsch. 27, 275-293, Stuttgart 1936.

Steeger, A.: Das glaziale Diluvium des Niederrheinischen Tieflandes. Beitr. VIII. Zur Frage einer Grundmoräne auf der linksrheinischen Mittelterrasse. - Ber. Vers. Niederrhein. Geol. Ver. f. 1925, Bonn.

- : Diluviale Bodenfrosterscheinungen am Niederrhein. - Diluvialgeologie u. Klima, hrsg. v. C. Troll. Geol. Rdsch., Bd. 34, H. 7/8, 520-538, Stuttgart 1944.

Tноме, K. N.: Eisvorstoß und Flußregime an Niederrhein und Zuider See im Jungpleistozän. Fortschr. Geol. Rheinl. u. Westf., Bd. 4, 197-246, Krefeld 1959.

Troll, C.: Strukturböden, Solifluktion und Frostklimate der Erde. - Diluvialgeologie u. Klima, hrsg. v. C. Troll. Geol. Rdsch., Bd. 34, H. 7/8, 545-694, Stuttgart 1944.

WAGNER, E.: Das niederrheinische Tiefland. Landeskundlicher Überblick. - Topographischer Atlas Nordrhein-Westfalen, Landesvermessungsamt NRW, o.O., 1968, S. 208.

Washburn, A. L.: Periglacial Processes and Environments. - London 1973.

Manuskript eingeg. 30. 7. 1974

Anschrift der Verf.: Dr. W. Golte und Prof. Dr. K. Heine, Geographisches Institut der Universität Bonn, 53 Bonn, Franziskanerstraße 2. 